\title{
Does our legal minimum drinking age modulate risk of first heavy drinking episode soon after drinking onset? Epidemiological evidence for the United States, 2006-2014
}

Hui G Cheng, James C. Anthony

Background: State-level 'age 21 ' drinking laws conform generally with the United States National Minimum Drinking Age Act of 1984 (US), and are thought to protect young people from adverse drinking experiences such as heavy episodic drinking (HED, sometimes called 'binge drinking'). We shed light on this hypothesis while estimating the age-specific risk of transitioning from $1^{\text {st }}$ full drink to $1^{\text {st } H E D ~ a m o n g ~ 12-t o-23-y e a r-o l d ~ n e w l y ~ i n c i d e n t ~}$ drinkers, with challenge to a "gender gap" hypothesis and male excess described in HED prevalence reports. Methods: The study population consisted of non-institutionalized civilians in the United States, with nine independently drawn nationally representative samples of more than 40,000 12-to-23-year-olds (2006-2014). Standardized audio computer-assisted self-interviews identified $\sim 43,000$ newly incident drinkers (all with $1^{\text {st }}$ HED evaluated within 12 months of drinking onset). Estimated age-specific HED risk soon after first full drink is evaluated for males and females. Results: Among 12-to-23-year-old newly incident drinkers, an estimated $20-30 \%$ of females and $35-45 \%$ of males experienced their $1^{\text {st }}$ HED within 12 months after drinking onset. Before mid-adolescence, there is no male excess in such HED risk. Those who postponed drinking to age 21 are not spared (27\% for 'postponer' females; $95 \% \mathrm{Cl}=24 \%$, 30\%; $42 \%$ for 'postponer' males; $95 \%$ $\mathrm{Cl}=38 \%, 45 \%)$. An estimated $10-18 \%$ females and $10-28 \%$ males experienced their $1^{\text {st }}$ HED in the same month of their $1^{\text {st }}$ drink; peak HED risk estimates are $18 \%$ for 'postponer' females $(95 \% \mathrm{Cl}=15 \%, 21 \%)$ and $28 \%$ for 'postponer' males $(95 \% \mathrm{Cl}=24 \%, 31 \%)$.

Conclusions: In the US, one in three young new drinkers transition into HED within 12 months after first drink. Those who postpone the $1^{\text {st }}$ full drink until age 21 are not protected. Furthermore, 'postponers' have substantial risk for very rapid transition to HED. A male excess in this transition to HED is not observed until after age 14. 
1

2

3

4

5

6

7

8

9

10

11

12

13

14

15

17

18

19

20

Manuscript under review; please do not cite or quote before published

25 May 2016

Does our legal minimum drinking age modulate risk of first heavy drinking episode soon after drinking onset? Epidemiological evidence for the United States, 2006-2014

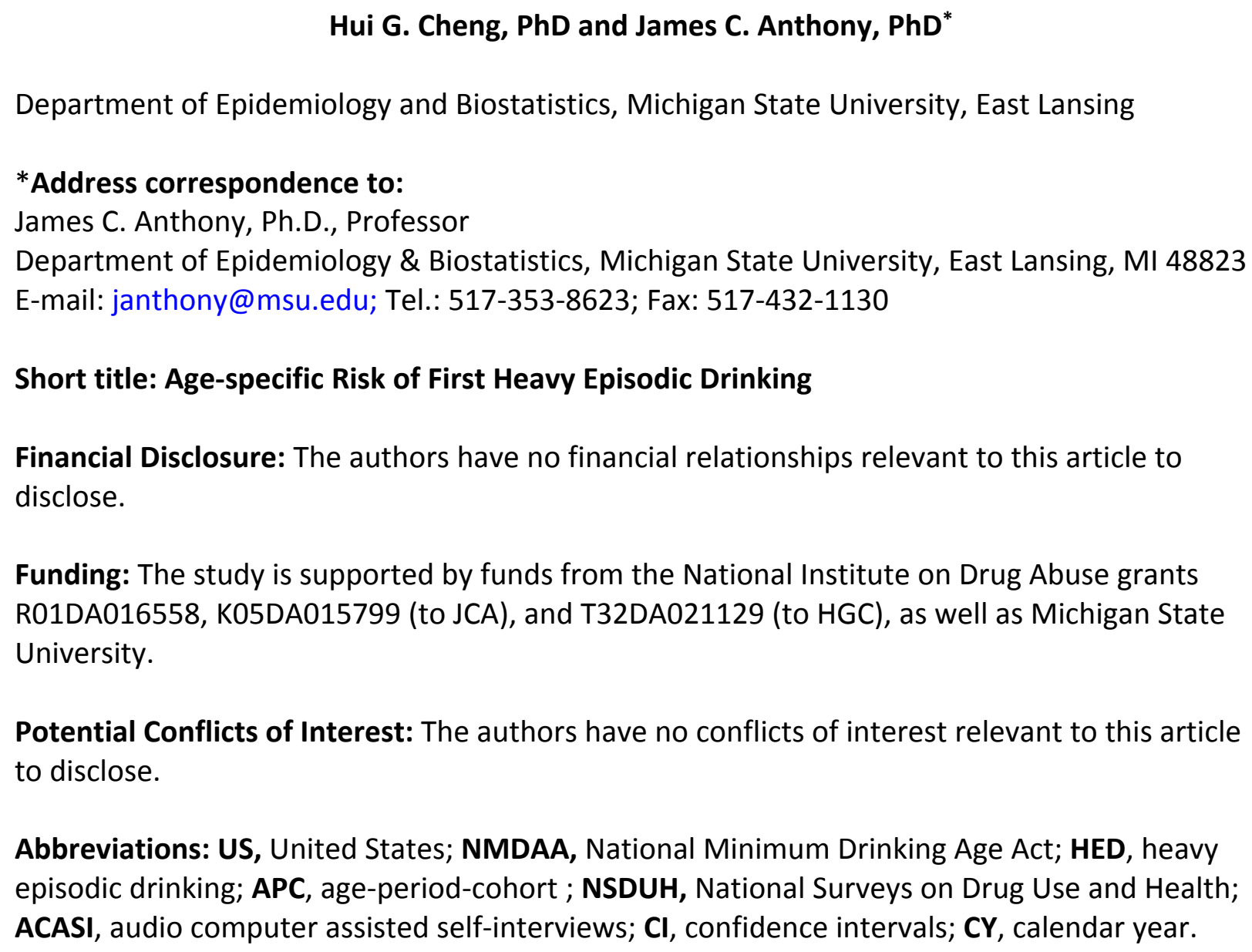

Potential Conflicts of Interest: The authors have no conflicts of interest relevant to this article to disclose.

Abbreviations: US, United States; NMDAA, National Minimum Drinking Age Act; HED, heavy episodic drinking; APC, age-period-cohort ; NSDUH, National Surveys on Drug Use and Health; ACASI, audio computer assisted self-interviews; $\mathrm{Cl}$, confidence intervals; $\mathbf{C Y}$, calendar year. 


\begin{abstract}
Background: State-level 'age 21' drinking laws conform generally with the United States National Minimum Drinking Age Act of 1984 (US), and are thought to protect young people from adverse drinking experiences such as heavy episodic drinking (HED, sometimes called 'binge drinking'). We shed light on this hypothesis while estimating the age-specific risk of transitioning from $1^{\text {st }}$ full drink to $1^{\text {st }}$ HED among 12-to-23-year-old newly incident drinkers, with challenge to a "gender gap" hypothesis and male excess described in HED prevalence reports.
\end{abstract}

Methods: The study population consisted of non-institutionalized civilians in the United States, with nine independently drawn nationally representative samples of more than 40,000 12-to23-year-olds (2006-2014). Standardized audio computer-assisted self-interviews identified 43,000 newly incident drinkers (all with $1^{\text {st }}$ HED evaluated within 12 months of drinking onset). Estimated age-specific HED risk soon after first full drink is evaluated for males and females.

Results: Among 12-to-23-year-old newly incident drinkers, an estimated $20-30 \%$ of females and $35-45 \%$ of males experienced their $1^{\text {st }}$ HED within 12 months after drinking onset. Before mid-adolescence, there is no male excess in such HED risk. Those who postponed drinking to age 21 are not spared (27\% for 'postponer' females; 95\% Cl=24\%, 30\%; 42\% for 'postponer' males; $95 \% \mathrm{Cl}=38 \%, 45 \%)$. An estimated $10-18 \%$ females and $10-28 \%$ males experienced their $1^{\text {st } H E D}$ in the same month of their $1^{\text {st }}$ drink; peak HED risk estimates are $18 \%$ for 'postponer' females $(95 \% \mathrm{Cl}=15 \%, 21 \%)$ and $28 \%$ for 'postponer' males $(95 \% \mathrm{Cl}=24 \%, 31 \%)$.

Conclusions: In the US, one in three young new drinkers transition into HED within 12 months after first drink. Those who postpone the $1^{\text {st }}$ full drink until age 21 are not protected. Furthermore, 'postponers' have substantial risk for very rapid transition to HED. A male excess in this transition to HED is not observed until after age 14. 


\section{Introduction}

Evaluated in the United States (US) and elsewhere in the world, adolescent and young adult drinkers are at increased risk of car crashes, drowning, and a broad range of other adverse events, as compared to same-cohort peers who do not drink. These drink-related adversities now help account for substantial alcohol-attributable disease burdens and social costs (Rehm et al. 2010; Spear \& Swartzwelder 2014). There are additional risks for adolescents who start drinking before late adolescence, and for those who make a fairly rapid transition into heavy episodic drinking (HED), usually defined as five or more drinks in one occasion (Crego et al. 2009; Crews et al. 2007).

\section{Consistent with a federal initiative intended to reduce HED and other harms from underage} drinking, in most states of the US, the legal minimum age for purchase and autonomous consumption of alcoholic beverages has been set at age 21 years. These 'age 21 ' laws are based on guidelines in the US National Minimum Drinking Age Act of 1984 (NMDAA).

At present, roughly three decades after NMDAA enactment, public health effects of the age 21 threshold continue to be debated (DeJong \& Blanchette 2014; Pitts et al. 2014; Wechsler \& Nelson 2010). Some US college presidents, among others, believe that the 'age 21 ' law in the US deserves reconsideration, and express concerns about unhealthy college campus impact of the 'age 21 ' laws, including what they observe about underage HED among college students (Rutledge et al. 2008). Nonetheless, a general societal expectation is optimistic, with benefits of the 'age 21 minimum' anticipated in the form of lower rates of unhealthy drinking behaviors, 
80

81

82

83

84

85

86

87

88

89

90

91

92

93

94

95

9

economic cost reductions, and improved public safety (DeJong \& Blanchette 2014; Prevention 2014; Wechsler \& Nelson 2010).

There may be an evidence-based rationale for setting a minimum legal minimum age for drinking alcohol, for smoking tobacco, and for other drug use, seen in estimates from epidemiological studies of these behaviors. These estimates often suggest disadvantageous consequences of early onsets and apparent benefits of delayed onsets. Disadvantages are most prominent among US subgroups with early drinking onsets during adolescence, judged by many to be a centrally important developmental stage when adolescents make major progress in brain maturation and toward acquisition of complex social skills (Hall et al. 2016; Schuckit 2009). In this context, we looked for opportunities to study newly incident underage drinkers and 'postponers' who have delayed their $1^{\text {st }}$ drink to age 21 , estimating age-specific risks of experiencing a $1^{\text {st }}$ heavy drinking episode soon after drinking onset (i.e., within 12 months after first full drink). We characterize this transition into HED (within 12 months) as a 'rapid-onset HED' based on prior studies (Reboussin \& Anthony 2006). In addition, recently completed survival analyses disclose a three years median induction interval for HED after drinking onset among newly incident US drinkers (i.e., lag time from age of first full drink to first HED occasion is $\geq 3$ years for $\geq 50 \%$ of drinkers; Cheng et al. Under review).

Our guiding hypothesis included an optimistic expectation of lower HED transition probabilities when adolescent males and females postpone their $1^{\text {st }}$ drink until age 21 years, as compared with larger expected values when corresponding risk estimates are made for underage drinkers. 
102 That is, we thought 'postponers' might have modulated (dampened) HED transition

103 probabilities in this comparison, in a reflection of (i) assumed benefits of prevailing 'age 21'

104 laws, as well as (ii) possibly greater pre-drink vulnerability to maladaptive drinking patterns in

105 subgroups of non-postponing underage drinkers as compared with subgroups of postponers.

107 As background, we note that almost all prior epidemiological HED estimates address the

'prevalence' of being a heavy episodic drinker, with few estimates about the 'incidence rates' or risk of experiencing the first HED soon after drinking starts. At present, sharply increasing ageassociated gradients in HED prevalence can be seen, with estimated $6 \%$ prevalence for $8^{\text {th }}$.

111 graders, $22 \%$ for $12^{\text {th }}$-graders, and even larger estimates for $20-24$ year olds. There also is a

112 general male excess in HED prevalence that might be narrowing (Patrick \& Schulenberg 2010;

113 Patrick et al. 2013).

115 Based on principles of epidemiological analysis, at population steady state, prevalence 116 proportions for a condition vary as the product of the 'incidence rate' for the condition times

117 the mean duration or persistence of the condition once it starts. In consequence, prior HED

118 studies that have estimated prevalence proportions leave us with an inherent ambiguity about

119 incidence processes versus persistence or duration-sustaining processes. In this instance, 120 incidence rates convey results of the process of 'becoming' affected (by HED) for the first time,

121 whereas prevalence proportions convey the state of 'being' affected (by HED). Estimates from

122 this study are distinctive and differ from prevalence study estimates because our focus is on 
123 'becoming' a heavy episodic drinker for the first time relatively soon after the onset of first full

124 drink.

125

126 Given this background, our study focuses on age-specific HED transitions observed quite soon

127 after $1^{\text {st }}$ drinks for 'postponers' who delay drinking onset to age 21 versus 'non-postponer'

128 underage drinkers. Intrigued by previously reported drinking games at $21^{\text {st }}$ birthday parties and

129 other occasions of the $1^{\text {st }}$ drink, we also estimate age-specific risks of very rapid transition to

130 HED such that $1^{\text {st }}$ drink and $1^{\text {st }}$ HED occur in the same month, which might be the birthday

131 month or other celebration of a person's $1^{\text {st }}$ drink (Rutledge et al. 2008). Given the possibility of

132 a narrowing 'gender gap' in HED, separate male and female estimates are provided in figures

133 and tables. A 'epidemiological mutoscope' view of individual cohort experiences is gained by

134 tracing estimates in the diagonal cells of these tables, as explained elsewhere (Cheng et al.

135 2016a; Cheng et al. 2016b; Seedall \& Anthony 2015). Whereas refinement of $21^{\text {st }}$ century

136 'epidemiological mutoscope' approaches has been described previously (Cheng et al. 2016a;

137 Seedall \& Anthony 2015), here we note that the mutoscope has its origins in research on

138 tuberculosis death rates conducted by the late Professor Wade Hampton Frost and in studies of

139 cognitive development by Warner Shaie (Frost 1939; Schaie 1977).

140

141 2. Methods

\section{$142 \quad 2.0$ Case Definitions for Heavy Episodic Drinking}

143 To start our methods section, we wish to bring the reader's attention to our deliberate and

144 reasoned calibration of our HED case definition, setting a criterion threshold at five drinks per 
145 occasion when studying 'postponers' versus 'non-postponers' and when studying male-female

146 differences. Our reasoning is based on a standard epidemiological research principle that case

147 definitions for incidence rates must first be held constant in order to discover whether there is

148 subgroup variation in incidence rates, with methods-related variations kept to minimum values.

149 This principle applies even though (1) 'postponers' to age 21 drinking onsets have more

150 autonomous freedom and a legal right to buy multiple drinks once the $21^{\text {st }}$ birthday anniversary

151 occurs, whereas availability and supply of multiple drinks might be more constrained for 'non-

152 postponer' underage drinkers, and (2) in research on adverse health effects of drinking, there is

153 one set of dose (i.e., drink)-response curves for females and a tendency for a rightward-shifted

154 dose-response curve for males (e.g., due to female-male variations in ethanol metabolism,

155 biotransformation, disposition, and excretion). This rightward-shift has motivated use of a 4+

156 drink threshold for females and a 5+ drink threshold for males in some prior HED research. In

157 this study, if we were to set a '4+ drink' threshold for non-postponers or for females, with a '5+

158 drink' threshold for postponers or for males, we would distort the initial comparisons, making it

159 necessary to repeat the subgroup analyses with calibration to the same threshold in order to

160 hold constant this methods-related source of variation in the incidence rates. By standardizing

161 the ' $5+$ drink' threshold for all subgroups, these initial estimates speak more clearly to the

162 question of whether postponers to age 21 are more or less likely to make the rapid transition

163 into HED, and to the question of whether males or females are more or less likely to do so. As

164 described below, the data are readily available so that other research teams can pursue this

165 line of research and can set alternative thresholds in future investigations (e.g., 4+ drinks for

166 females versus $5+$ drinks for males) 
168 We also make a note about our 'mutoscope' approach of tracing the alcohol experience of each 169 cohort down the diagonals of our tables (age by age, and year by year), in a fashion that allows 170 one to check whether the forward progress of a cohort (as shown in the table diagonals) is or is

171 not congruent with the pattern of age-specific estimates (as shown in the table rows). As

172 explained elsewhere, this mutoscope approach assumes a null or negligible 'period effect' and

173 is not the same as an age-period-cohort (APC) analysis (Cheng et al., 2016). Nonetheless, for

174 interested readers, we provide results from an APC analysis of these data, as explained in 175 Section 2.3.

176

$177 \quad 2.1$ Study population, sample, and assessments

178 The study population is defined to include non-institutionalized community dwelling unit 179 residents aged $12+$ years in the US, as studied between 2006 and 2014. Estimates for this study population are from nationally representative samples drawn for the US National Surveys on

Drug Use and Health (NSDUH), with annual re-sampling and standardized confidential in-person assessments after IRB-approved parent consent and child assent; detailed methods descriptions are provided elsewhere (Seedall \& Anthony 2015; United States 2012; United States 2015). Participation levels range from $72 \%$ to $76 \%$, yielding more than 250,00012 -to-23 year olds. We did not provide estimates for individuals older than 23 years of age because few people in the

186 US start drinking after age 23 years (Cheng et al. 2016a; Cheng et al. 2016b). 
188 The NSDUH confidential assessments were conducted as audio computer assisted self-

189 interviews (ACASI), each with standardized multi-item modules on health and drugs, including

190 alcohol. Items assessed month, year, and age of $1^{\text {st }}$ full drink and of $1^{\text {st }}$ HED $(5+$ drinks on a

191 single occasion), and identified >24,000 12-to-23-year-olds who qualified as 'newly incident

192 drinkers,' with $1^{\text {st }}$ drinking onset within 12 months before assessment, but with no prior

193 drinking of one full drink before that interval. (NSDUH survey data exist from 2002-2014, but

194 information about the date of the $1^{\text {st }}$ HED is available only since 2006.) The final analytic sample

195 size is 24,100 12-to-23-year-old newly incident drinkers (mean age of drinking onset $=16.5$ ).

196 Supplementary table S1 provides a description of unweighted sizes of the samples from which

197 these newly incident drinkers were drawn.

198

199

Due to confidentiality concerns, the exact date of interview and birthdates are not available in 200 publicly downloadable NSDUH datasets. Instead, only the quarter of the year when the

201

202

203

204

205

206

207

208

209

interview was conducted is available. Therefore, cases of newly incident drinking consist of

those who initiated drinking within the past four quarters prior to the assessment, with at least one drink in the 12 months prior to assessment. A similar method was used to identify newly incident HED. Newly incident HED cases had $1^{\text {st }}$ HED within the same 12 months as $1^{\text {st }}$ drink.

\subsection{Analysis approach}

Each age-specific transition probability from $1^{\text {st }}$ consumption of a full drink to $1^{\text {st }}$ HED among newly incident drinkers was conceptualized with an analysis-weighted numerator consisting of newly incident HED cases arising from an analysis-weighted denominator of newly incident 
210 drinkers who initiated drinking at a specific age, all events conceptualized to occur within 12

211 months of the assessment date, including $1^{\text {st }}$ HED occurrences. In this study, we defined rapid

212 transition as the first HED occurring within 12 months of drinking onset, which is consistent

213 with our previous research on drug-related outcomes (Reboussin \& Anthony 2006;

214 Vsevolozhskaya \& Anthony 2015). [We note that NSDUH discloses assessment quarter (but not

215 assessment month). For this reason, the interval for estimation of HED transition probabilities

216 after $1^{\text {st }}$ drink typically is 12 months, but might be as large as 15 months for a small fraction of

217 newly incident drinkers.]

218

219 Analogously, for 'very rapid' transition from $1^{\text {st }}$ drink to $1^{\text {st }} \mathrm{HED}$, the transition probability was

220 conceptualized with an analysis-weighted numerator consisting of newly incident HED cases

221 whose $1^{\text {st }}$ drink and $1^{\text {st }}$ HED occurred in the same month arising from an analysis-weighted

222 denominator of newly incident drinkers who initiated drinking within 12 months of the

223 assessment date. [In these analyses, the month of $1^{\text {st }}$ full drink is the same as the month of $1^{\text {st }}$

224 HED, and the $1^{\text {st }}$ HED might have been on the same day as the $1^{\text {st }}$ full drink.]

225

226 All estimates are based on NSDUH year-specific analysis weights that account for sample

227 selection probabilities and post-stratification adjustment factors to replicate US Census

228 subpopulation counts. Row-wise, the tabled cells depict age-specific patterns. The mutoscope

229 view is gained by reading the same table cells down the diagonals in evaluation of whether

230 cohort-specific patterns are congruent with age-specific patterns. Standard errors and 95\%

231 confidence intervals $(\mathrm{Cl})$ are from complex survey delta methods. 
233 Next, logarithms were taken and meta-analysis summaries of age-specific estimates were 234 derived from the nine independent replications. Random-effects estimators were used when 235 heterogeneity was detected (DerSimonian \& Laird 1986; Higgins et al. 2003). As a check on an assumption about null or negligible 'period effects' in the interpretation of the mutoscope view,

237 an APC analysis approach known as 'constrained regression' was used (Harper 2015). The 238 constrained regression approach solves an APC problem of model identification via equality constraints. Here, we applied a theoretically plausible (CY2013=CY2014) period constraint. More details about the use of meta-analysis and APC in the 'mutoscope' approach is provided

241 in a previous publication (Cheng et al. 2016a).

\section{Results}

Figure 1 shows meta-analysis summary estimates for rapid-onset HED transition probabilities with the age at $1^{\text {st }}$ full drink on the $x$-axis, and a one year interval of observation after that drink. For males, one of the estimated peak values in risk of transitioning from $1^{\text {st }}$ drink to $1^{\text {st }}$ HED within that year is seen among 'postponers' with age 21 drinking onset (Males age 21: $42 \% ; 95 \% \mathrm{Cl}=38 \%, 45 \%)$. This $42 \%$ transition probability estimate is not too distant from expected values based on 'non-postponer' underage male drinkers in mid-late adolescence. adolescence (i.e., 14 years of age and younger). 
253 Among female 'postponer' newly incident drinkers who delayed $1^{\text {st }}$ drink to age 21 , the

254 estimated risk of experiencing a HED within one year after $1^{\text {st }}$ drink is $27 \%(95 \% \mathrm{Cl}=24 \%, 30 \%)$.

255 This HED risk estimate is substantially smaller than the comparably observed male estimate at

256 age 21 , but is not appreciably different from the peak value among 'non-postponer' underage

257 female drinkers in mid-late adolescence (e.g., Figure 1 Females age 18: 29\%; 95\% Cl=27\%, 32\%).

258 Among female newly incident drinkers observed within one year after $1^{\text {st }}$ full drink, HED risk

259 does not vary robustly with age of 1st full drink. A robust male excess in these HED risk

260 estimates can be seen from age 15 onward, but not when underage drinking starts at age 11-to-

261 14-years.

262

263

A different pattern is seen when we shift the focus to very rapid transition to HED (i.e., $1^{\text {st }}$ drink and $1^{\text {st }}$ HED occurring in the same month, Figure 1$)$. In a comparison with underage new drinkers, the risk of this "very rapid" transition to HED is much higher among 'postponers' for both males and females (males: $28 \%, 95 \% \mathrm{Cl}=24 \%, 31 \%$; females: $18 \%, 95 \% \mathrm{Cl}=15 \%, 21 \%$ ). The corresponding estimates are $\sim 20 \%$ and $\sim 12 \%$ for late-adolescent newly incident male and female drinkers, respectively.

Viewed along the mutoscopic trace down the diagonal cells, Table 1 discloses cohort-specific

271 transitions through adolescence and into the early 20s, based upon re-sampling of each cohort

272 from 2006 through 2014. (Table 2 shows the 95\% confidence intervals for the estimated

273 transition probabilities.) In Table 1, for males, as we look down the diagonals from age 16 years 
274 in 2006 to age 21 years in 2011, we see the peak HED risk estimate at age 21 years. A similar

275 pattern is seen for other male cohorts transitioning through age 21 years.

276

277 A generally congruent pattern can be seen via the mutoscope trace of diagonals for newly

278 incident female drinkers, age by age, and year by year. For very rapid transition to HED, the

279 diagonals of Table 3 reveal a highly consistent peak at 21 in all cohorts that traverse age 21

280 years, for females as well as for males.

281

282

To complement the just-reported HED transition probabilities for newly incident drinkers, a set

of age-specific unconditional HED population incidence rates for males and females was

estimated by re-specifying denominators to include these additional subgroups: (1) all never

drinkers at each age, and (2) all past-onset drinkers with no HED experience at each age - as

shown in supplementary Tables S1-2. In year-specific and meta-analysis form, these annual

unconditional HED population incidence rates are quite low at age 12 years (female: age 12:

values at age 21 years for both females and males (female: $28.7 \%$; $95 \% \mathrm{Cl}=27.1 \%, 30.3 \%$; male:

$33.6 \%$; $95 \% \mathrm{Cl}=31.8 \%, 35.5 \%)$. There is no apparent male-female difference in these

unconditional HED population incidence rates prior to age 17 (supplementary Figure S1).

The constrained regression models confirmed our assumption of no tangible 'period effects' in

294 either set of estimates. With age and period 'effects' held constant, the estimated 'cohort 
295 effects' were null. Within the APC framework, with cohort and period 'effects' held constant,

296 the age pattern is congruent with what is described above.

297

298

\section{Discussion}

299

What have we discovered? First, studied in aggregate, more than one in three of the US

300

adolescent and young adult newly incident drinkers transition rapidly to HED (20-30\% in

301

females and $30-45 \%$ in males). Second, for both males and females, the 'postponers' who do

302

not drink until age 21 years represent a subgroup of newly incident drinkers who are most likely

to engage in heavy episodic drinking during the month of the $1^{\text {st }}$ full drink (Figure 1 , Panel 2 ). In

addition, 'postponement' of $1^{\text {st }}$ drink to the NMDAA-encouraged age 21 threshold does not seem to modulate risk of transitioning into a rapid-onset HED within 12 months after the $1^{\text {st }}$

drink, nor does it provide protection against HED risk, when the comparison group consists of

newly incident underage drinkers. These general patterns can be seen in age-specific HED

transition probability estimates for both sexes, and also in mutoscopic diagonal traces of each

before mid-adolescence. From age 15 years onward, newly incident male drinkers are more

likely than newly incident female drinkers to show rapid onset of $1^{\text {st }}$ HED. This pattern can be seen most clearly in Figure 1.

As for limitations and counterbalancing strengths, NSDUH relies on self-reports from 12-to-23year-olds in community survey samples, but is not restricted to school attendees, and therefore

316 should be more generalizable than estimates based on school surveys. In addition, these HED 
317 risk estimates lack fine-grained time specifications (e.g., exact dates for birthdate, $1^{\text {st }}$ drink, $1^{\text {st }}$

318 HED), and do not address a long-term cumulative HED risk as might be seen years after the $1^{\text {st }}$

319 drink. The NSDUH data are not entirely appropriate for research on cumulative HED incident

320 proportions or HED hazard estimates beyond the first 12-24 months after newly incident

321 drinking. It is possible that the postponers to age 21 experience an unexceptional transition

322 probability during the $1^{\text {st }}$ year after $1^{\text {st }}$ drink, after which the conditional HED risk drops

323 markedly such that the cumulative incidence proportion is not appreciably greater than the

324 corresponding proportion for a more rapid transition into HED. In contrast, the risk of first HED

325 might be distributed over the first 5-9 years of drinking for those who start drinking at age 11-

326 14, such that there is a more gradual accumulation of HED risk, possibly ending up with a

327 cumulative incidence proportion for non-postponer underage drinkers versus postponer 'age

$32821^{\prime}$ drinkers. This open question can be resolved via life table analyses to estimate both

329 'instantaneous' hazard of HED after the $1^{\text {st }}$ drink as well as the 'cumulative' incidence

330 proportions across multiple years of experience after the $1^{\text {st }} \mathrm{drink}$.

332 An important counterbalancing strength of this study can be seen in its multiple independent replications to ensure reproducibility. In addition, the large nationally representative samples and use of ACASI boost external and internal validity.

We also note that rapid-onset HED is the only unhealthy drinking consequence studied here.

337 This study's evidence does not speak to whether delay of the $1^{\text {st }}$ drink to age 21 has other

338 public health benefits, as might be seen in modulation of risk (or prevalence) of other 
consequences of importance, such as driving under the influence of alcohol (DeJong \&

Blanchette 2014; Wechsler \& Nelson 2010).

341

342 Notwithstanding considerations such as these, the discovery that $30-40 \%$ of newly incident drinkers aged 12 -to-23-years-old transition from $1^{\text {st }}$ full drink to $1^{\text {st }}$ HED within roughly one year

344 after $1^{\text {st }}$ full drink might be a noteworty finding for alcohol epidemiology in the US, with

345 implications for future cross-national research when there is no 'age 21 ' legal minimum. Even

346 though we do not see major age-related differences in HED risk, earlier adolescent drinkers may

347 be particularly vulnerable to cumulative HED risk, as noted above, or to other adverse drinking

348 consequences (Crego et al. 2009; Crews et al. 2007; Plunk et al. 2014; Rehm et al. 2010; Spear \&

349 Swartzwelder 2014). The findings throw light on public health actions to prevent HED even

350 when underage drinking has not been prevented, perhaps involving linkages or joint efforts of

351 clinicians, schools, families, and community outreach programs

353 This study's estimates run contrary to our expectation of HED risk modulation via

354 postponement of $1^{\text {st }}$ drink to the NMDAA-encouraged drinking age threshold at 21 years. The

355 resulting evidence deserves consideration in a more thorough discussion of alternatives to the

356 age 21 threshold (Brister et al. 2010; Lewis et al. 2009; Rutledge et al. 2008; Wechsler \& Nelson

357 2010). Similarly, our findings about a very rapid transition to HED stand in stark contrast with

358 our hypothesis about lower risks of HED in 'postponer' males and females. Indeed, we found

359 that approximately $30 \%$ of 'postponer' males and $20 \%$ of 'postponer' females transition to HED

360 within the same month of drinking onset, the highest among all age groups studied. Although 
361 we do not have a solid theory or explanation for this observation, we consider a possibility that

362 previously described $21^{\text {st }}$ birthday drinking games, or non-birthday celebrations of the $1^{\text {st }}$ drink,

363 might play an important role (Rutledge et al. 2008). Subject to confirmation and elaboration in

364 future studies, our findings highlight the importance of effective prevention strategies intended

365 to discourage the initiation of drinking games with the first year after drinking onset, including

366 birthday games (Rutledge et al. 2008).

367

368 In this study, among 12-to-14-year-olds, we found no clear male-female differences in

369 estimates for making transitions into HED within roughly one year after $1^{\text {st }}$ full drink. This

370 finding is in line with previous studies documenting an absence of a 'gender gap' in alcohol

371 consumption among early adolescence (Schulte et al. 2009), including recently published

372 evidence of a female excess risk of starting to drink before the $18^{\text {th }}$ birthday in recent US

373 cohorts (Seedall \& Anthony, 2013). This study's observed male excess in HED from age 15

374 onward most likely has maturational, social, and biological origins that deserve more study

375 (Keyes et al. 2008; Kuhn 2015; Kuntsche et al. 2015; Schwartz 2013; Seedall \& Anthony 2015).

376 Future studies can inspect physiological changes coincident with the emergence of risk-taking

377 behaviors, as well as learned social roles and expectations, in order to clarify underlying

378 mechanisms for this observed age-specific HED risk variation, which might involve androgens or

379 emergence of gender-related social identities (Byrnes et al. 1999). In these future studies,

380 facets of the general "gender gap" can be explored usefully by comparing HED estimates based

381 on two approaches: (1) the calibrated approach used here, with the same HED case definition

382 for both sexes, and (2) the sex-differentiated HED metric as can be motivated when the primary 
383

384 (Wilsnack et al. 2000).

\section{Conclusion}

We conclude on a theoretical note with potential clinical practice relevance. Suppose young people who postpone drinking onsets until the $21^{\text {st }}$ birthday truly are at modestly excess HED teams have commented upon these " $21^{\text {st }}$ birthday drinking virgins," noting that some of them consume too much alcohol as part of the ritual celebration of this transition into adult privileges (Brister et al. 2010; Lewis et al. 2009; Neighbors et al. 2009; Rutledge et al. 2008). We theorize that it is not age 21 per se that is crucial. Rather, the important experience might be the rite of passage into drinking on one's own, whether at age 21 years, or in earlier teen 395 years. To be sure, parents, teachers, and clinicians seeing 20 year olds on the approach to the $21^{\text {st }}$ birthday might try a brief intervention in an attempt to shape the nature of that ritual experience and to dampen its potentially unhealthy facets. Nevertheless, these same brief interventions can be used by parents, teachers, and pediatricians who ask about and discover adolescents who have just started 'drinking on their own,' perhaps with deliberate adaptation of the interventions for younger audiences, and with a referral to a 'smartphone app' or other electronic technology program after the quick pediatric consult on this topic (Leeman et al. 2015; White et al. 2010). If we are correct, next steps should include more refinement of the increasingly appropriate 'quick pediatric consult' approach by medical practitioners or by school 404 counselors and teachers, plus parent-delivered brief intervention approaches. Ultimately, 
405 randomized clinical trials of promising refinements in these novel programs will be needed so

406 that their efficacy, effectiveness, and unanticipated externalities can be estimated before

407 widespread dissemination. 


\section{Acknowledgments}

409 The content is the sole responsibility of the authors and does not necessarily represent the

410 official views of MSU, the National Institute on Drug Abuse, or the National Institutes of Health.

411 The authors wish to thank the United States Substance Abuse and Mental Health Services

412 Administration Office of Applied Studies (now the Center for Behavioral Health Statistics and

413 Quality) for completion of its annual nationally representative surveys on drug use and health, 414 as well as its direction and supervision of the annual data gathering and preparation of public 415 use datasets. Authors are grateful for valuable advice and research assistance from Mr. Karl 416 Alcover, as well as helpful suggestions from Dr. Catalina Lopez-Quintero. 
418

419

420

421

422

423

424

425

426

427

428

429

430

431

432

433

434

435

436

437

438

439

440

441

442

443

444

445

446

447

448

449

450

451

452

453

454

455

456

457

458

459

460

461

462

463

464

\section{References}

Brister HA, Wetherill RR, and Fromme K. 2010. Anticipated versus actual alcohol consumption during 21st birthday celebrations. J Stud Alcohol Drugs 71:180-183.

Byrnes JP, Miller DC, and Schafer WD. 1999. Gender Differences in Risk Taking: A Meta-Analysis. Psychological Bulletin 125:7.

Cheng HG, Cantave MD, and Anthony JC. 2016a. Alcohol Experiences Viewed Mutoscopically: Newly Incident Drinking of Twelve- to Twenty-Five-Year-Olds in the United States, 2002-2013. Jouranl of Studies on Alcohol and Drugs 77:405-412.

Cheng HG, Cantave MD, and Anthony JC. 2016b. Taking the first full drink: Epidemiological evidence on male-female differences in the United States. Alcohol Clin Exp Res 40:816-825.

Cheng HG, Wetherington CL, and Anthony JC. Under Review. Male-Female Differences in the Onset of Heavy Episodic Drinking Soon after First Full Drink in Contemporary United States: From Early Adolescence to Young Adulthood.

Crego A, Holguin SR, Parada M, Mota N, Corral M, and Cadaveira F. 2009. Binge drinking affects attentional and visual working memory processing in young university students. Alcohol Clin Exp Res 33:1870-1879. 10.1111/j.1530-0277.2009.01025.x

Crews F, He J, and Hodge C. 2007. Adolescent cortical development: a critical period of vulnerability for addiction. Pharmacol Biochem Behav 86:189-199. 10.1016/j.pbb.2006.12.001

DeJong W, and Blanchette J. 2014. Case closed: research evidence on the positive public health impact of the age 21 minimum legal drinking age in the United States. J Stud Alcohol Drugs Suppl 75 Suppl 17:108-115.

DerSimonian R, and Laird N. 1986. Meta-analysis in clinical trials. Control Clin Trials 7:177-188.

Frost WH. 1939. The age selection of mortality from tuberculosis in successive decades. The American Journal of Hygiene:91-96.

Hall WD, Patton G, Stockings E, Weier M, Lynskey M, Morley KI, and Degenhardt L. 2016. Why young people's substance use matters for global health. The Lancet Psychiatry 3:265-279. 10.1016/s2215-0366(16)00013-4

Harper S. 2015. Invited Commentary: A-P-C ... It's Easy as 1-2-3! Am J Epidemiol 182:313-317. 10.1093/aje/kwv052

Higgins JP, Thompson SG, Deeks JJ, and Altman DG. 2003. Measuring inconsistency in meta-analyses. BMJ 327:557-560. 10.1136/bmj.327.7414.557

Keyes KM, Grant BF, and Hasin DS. 2008. Evidence for a closing gender gap in alcohol use, abuse, and dependence in the United States population. Drug Alcohol Depend 93:21-29. 10.1016/j.drugalcdep.2007.08.017

Kuhn C. 2015. Emergence of sex differences in the development of substance use and abuse during adolescence. Pharmacol Ther 153:55-78. 10.1016/j.pharmthera.2015.06.003

Kuntsche E, Wicki M, Windlin B, Roberts C, Gabhainn SN, van der Sluijs W, Aasvee K, Gaspar de Matos M, Dankulincova Z, Hublet A, Tynjala J, Valimaa R, Bendtsen P, Vieno A, Mazur J, Farkas J, and Demetrovics Z. 2015. Drinking motives mediate cultural differences but not gender differences in adolescent alcohol use. J Adolesc Health 56:323-329. 10.1016/j.jadohealth.2014.10.267

Leeman RF, Perez E, Nogueira C, and DeMartini KS. 2015. Very-Brief, Web-Based Interventions for Reducing Alcohol Use and Related Problems among College Students: A Review. Front Psychiatry 6:129. 10.3389/fpsyt.2015.00129

Lewis MA, Lindgren KP, Fossos N, Neighbors C, and Oster-Aaland L. 2009. Examining the relationship between typical drinking behavior and 21st birthday drinking behavior among college students: implications for event-specific prevention. Addiction 104:760-767. 10.1111/j.13600443.2009.02518.x 
465

466

467

468

469

470

471

472

473

474

475

476

477

478

479

480

481

482

483

484

485

486

487

488

489

490

491

492

493

494

495

496

497

498

499

500

501

502

503

504

505

506

507

508

509

510

511

512

Neighbors C, Lee CM, Lewis MA, Fossos N, and Walter T. 2009. Internet-based personalized feedback to reduce 21st-birthday drinking: a randomized controlled trial of an event-specific prevention intervention. J Consult Clin Psychol 77:51-63. 10.1037/a0014386

Patrick ME, and Schulenberg JE. 2010. Alcohol use and heavy episodic drinking prevalence and predictors among national samples of American eighth- and tenth-grade students. J Stud Alcohol Drugs 71:41-45.

Patrick ME, Schulenberg JE, Martz ME, Maggs JL, O'Malley PM, and Johnston LD. 2013. Extreme binge drinking among 12th-grade students in the United States: prevalence and predictors. JAMA Pediatr 167:1019-1025. 10.1001/jamapediatrics.2013.2392

Pitts JR, Johnson ID, and Eidson JL. 2014. Keeping the case open: responding to DeJong and Blanchette's "Case closed" on the minimum legal drinking age in the United States. J Stud Alcohol Drugs 75:1047-1049.

Plunk AD, Syed-Mohammed H, Cavazos-Rehg P, Bierut L, and Grucza RA. 2014. Alcohol consumption, heavy drinking, and mortality: rethinking the j-shaped curve. Alcohol Clin Exp Res 38:471-478. 10.1111/acer.12250

Prevention USCfDCa. 2014. Excessive Drinking Costs U.S. \$223.5 Billion. Available at http://www.cdc.gov/Features/AlcoholConsumption/(accessed October 15 2015).

Reboussin BA, and Anthony JC. 2006. Is there epidemiological evidence to support the idea that a cocaine dependence syndrome emerges soon after onset of cocaine use? Neuropsychopharmacology 31:2055-2064. 10.1038/sj.npp.1301037

Rehm J, Baliunas D, Borges GL, Graham K, Irving H, Kehoe T, Parry CD, Patra J, Popova S, Poznyak V, Roerecke M, Room R, Samokhvalov AV, and Taylor B. 2010. The relation between different dimensions of alcohol consumption and burden of disease: an overview. Addiction 105:817-843. 10.1111/j.1360-0443.2010.02899.x

Rutledge PC, Park A, and Sher KJ. 2008. 21st birthday drinking: extremely extreme. J Consult Clin Psychol 76:511-516. 10.1037/0022-006X.76.3.511

Schaie KW. 1977. Toward a stage theory of adult cognitive development. Int J Aging Hum Dev 8:129-138. Schuckit MA. 2009. Alcohol-use disorders. Lancet 373:492-501. 10.1016/S0140-6736(09)60009-X

Schulte MT, Ramo D, and Brown SA. 2009. Gender differences in factors influencing alcohol use and drinking progression among adolescents. Clin Psychol Rev 29:535-547. 10.1016/j.cpr.2009.06.003

Schwartz J. 2013. A "New" Female Offender or Increasing Social Control of Women's Behavior? CrossNational Evidence. Feminist Studies 39:790-821.

Seedall RB, and Anthony JC. 2015. Monitoring by Parents and Hypothesized Male-Female Differences in Evidence from a Nationally Representative Cohort Re-sampled from Age 12 to 17 Years: An Exploratory Study Using a "Mutoscope" Approach. Prev Sci 16:696-706. 10.1007/s11121-0140517-8

Spear LP, and Swartzwelder HS. 2014. Adolescent alcohol exposure and persistence of adolescenttypical phenotypes into adulthood: a mini-review. Neurosci Biobehav Rev 45:1-8. 10.1016/j.neubiorev.2014.04.012

United States. 2012. Substance Abuse and Mental Health Services Administration. Center for Behavioral Health Statistics and Quality. Comparing and evaluating youth substance use estimates from the National Survey on Drug Use and Health and other surveys (HHS Publication No. SMA 12-4727, Methodology Series M-9). Rockville, MD: Substance Abuse and Mental Health Services Administration.

United States. 2015. Department of Health and Human Services. Substance Abuse and Mental Health Services Administration. Center for Behavioral Health Statistics and Quality. National Survey on Drug Use and Health: 2-Year R-DAS (2002 to 2003, 2004 to 2005, 2006 to 2007, 2008 to 2009, 
Vsevolozhskaya OA, and Anthony JC. 2015. Transitioning from First Drug Use to Dependence Onset: Illustration of a Multiparametric Approach for Comparative Epidemiology. Neuropsychopharmacology. 10.1038/npp.2015.213 drinking age decrease drinking and related consequences among youths? Am J Public Health 100:986-992. 10.2105/AJPH.2009.178004

White A, Kavanagh D, Stallman H, Klein B, Kay-Lambkin F, Proudfoot J, Drennan J, Connor J, Baker A, Hines E, and Young R. 2010. Online alcohol interventions: a systematic review. J Med Internet Res 12:e62. 10.2196/jmir.1479

Wilsnack RW, Vogeltanz ND, Wilsnack SC, Harris TR, Ahlstrom S, Bondy S, Csemy L, Ferrence R, Ferris J, Fleming J, Graham K, Greenfield T, Guyon L, Haavio-Mannila E, Kellner F, Knibbe R, Kubicka L, Loukomskaia M, Mustonen H, Nadeau L, Narusk A, Neve R, Rahav G, Spak F, Teichman M, Trocki K, Webster I, and Weiss S. 2000. Gender differences in alcohol consumption and adverse drinking consequences: cross-cultural patterns. Addiction 95:251-265. 
Figure 1. Comparison of Meta-Analytic Summary Estimates for Sex- And Age-Specific Probability (\%) of Transitioning from $1^{\text {st }}$ Drink to $1^{\text {st }}$ Heavy Episodic Drinking Among Newly Incident Drinkers Who Started Drinking Within 12 Months Prior to the Assessment. Data From United States National Surveys on Drug Use And Health, 2006-2014. (Unweighted n =23,735 12-to-21year-olds) ${ }^{1}$

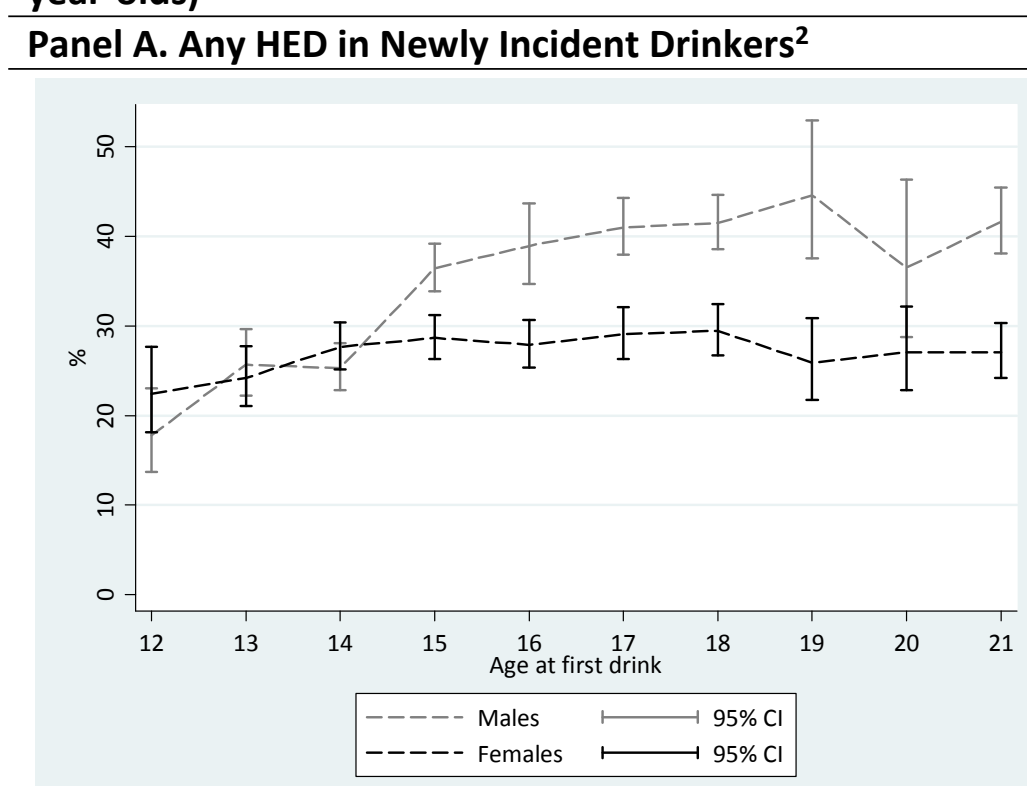
Panel B. Transition to HED Within the Same Month ${ }^{3}$

\footnotetext{
${ }^{1}$ Estimates for 22- and 23-year-olds are presented in Table 1 and 3, but are not shown due to suboptimal precision associated with the few newly incident drinkers observed in this sample after age 21 years.

2. For 16-, 19-, and 20-year old males, heterogeneity across replications motivated use of the random effects variance estimation approach.

3. For 12-, 13-, 18-, 19-, and 20-year old males as well as 17-year-old females, heterogeneity across replications motivated use of the random effects variance estimation approach.
}

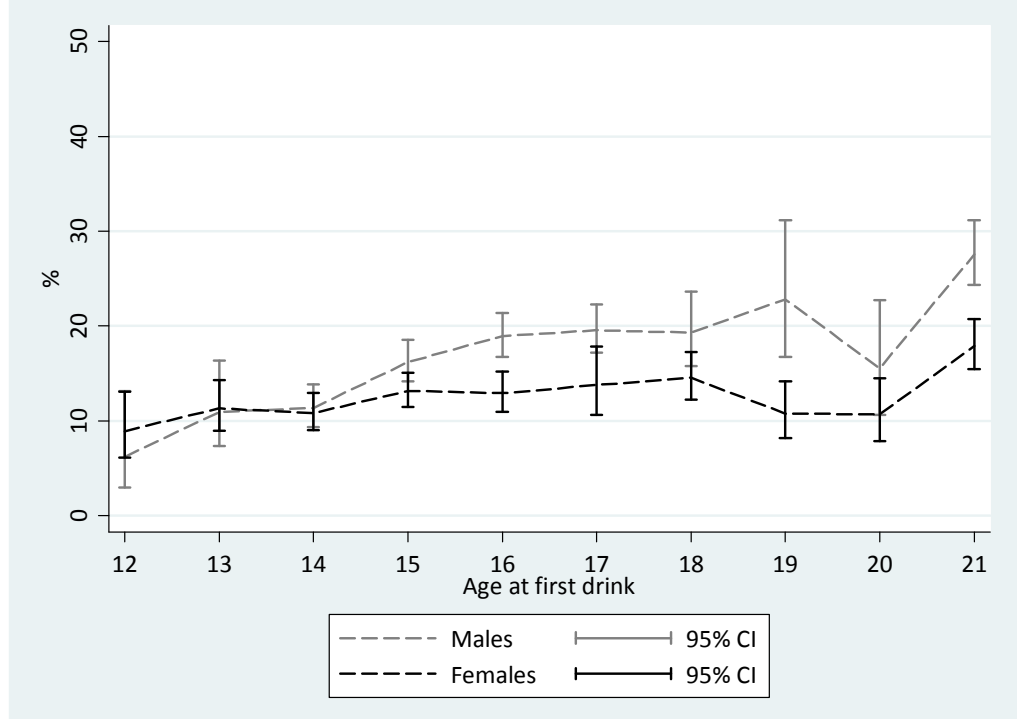




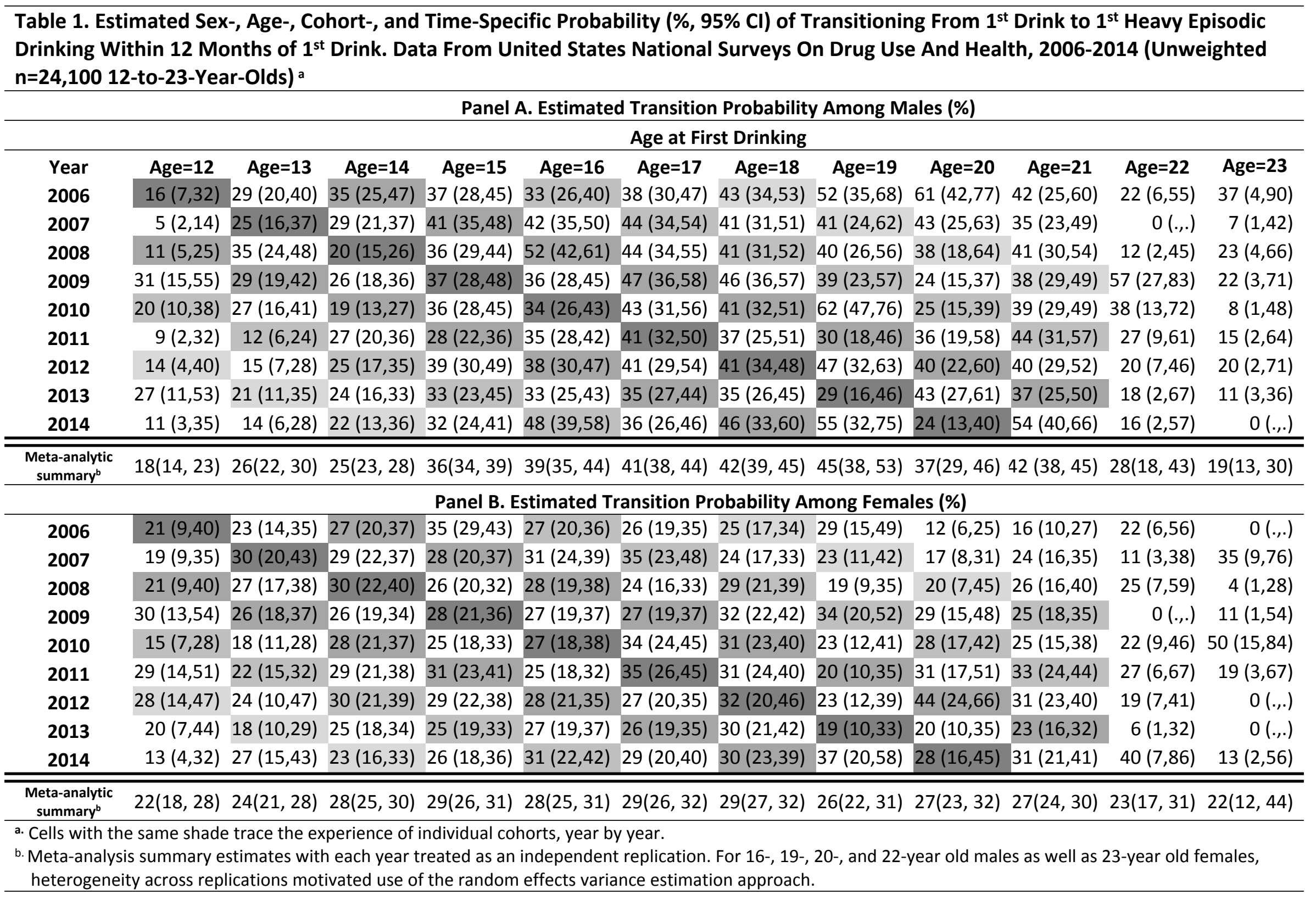


Table 2. Estimated Sex-, Age-, Cohort-, and Time-Specific Probability (\%, $95 \% \mathrm{Cl})$ of Transitioning From $1^{\text {st }}$ Drink to $1^{\text {st }}$ Heavy Episodic Drinking Within the Same Month of $1^{\text {st }}$ Drink. Data From United States National Surveys On Drug Use And Health, 2006-2014 (Unweighted $n=24,100$ 12-to-23-Year-Olds) ${ }^{a}$

\begin{tabular}{|c|c|c|c|c|c|c|c|c|c|c|c|c|}
\hline Year & \multicolumn{12}{|c|}{ Panel A. Estimated Transition Probability Among Males (\%) } \\
\hline 2006 & $9(3,25)$ & $16(9,26)$ & $16(8,28)$ & $16(10,24)$ & $17(11,25)$ & $22(16,30)$ & $21(13,31)$ & $28(16,46)$ & $33(18,52)$ & $25(13,41)$ & $20(4,56)$ & $0(.,)$. \\
\hline 2008 & $2(0,9)$ & $18(11,29)$ & $11(7,17)$ & $12(8,18)$ & $25(18,34)$ & $22(14,31)$ & $28(19,39)$ & $12(6,22)$ & $16(6,38)$ & $34(24,47)$ & $0(.,)$. & $0(.,)$. \\
\hline 2009 & $20(7,44)$ & $18(9,30)$ & $16(9,27)$ & $11(6,18)$ & $17(11,25)$ & $20(12,29)$ & $19(12,30)$ & $10(4,22)$ & $11(4,27)$ & $14(9,22)$ & $44(9,87)$ & $0(.,)$. \\
\hline 2010 & $3(1,14)$ & $3(1,11)$ & $10(5,18)$ & $17(11,27)$ & $12(8,20)$ & $19(12,30)$ & $20(13,30)$ & $41(29,53)$ & $7(2,23)$ & $31(22,42)$ & $38(13,72)$ & $8(1,48)$ \\
\hline 2012 & $0(.,)$. & $1(0,7)$ & $13(7,25)$ & $24(17,34)$ & $17(11,24)$ & $21(12,33)$ & $14(9,20)$ & $26(13,46)$ & $10(5,22)$ & $24(16,35)$ & $13(4,36)$ & $20(2,71)$ \\
\hline 2013 & $12(3,42)$ & $13(5,30)$ & $10(5,18)$ & $15(10,22)$ & $15(10,22)$ & $16(10,24)$ & $11(6,19)$ & $21(10,39)$ & $27(15,45)$ & $29(18,42)$ & $0(.,)$. & $0(.,)$. \\
\hline 2014 & $3(0,20)$ & $3(0,22)$ & $6(3,15)$ & $18(10,30)$ & $23(15,33)$ & $18(11,28)$ & $19(10,33)$ & $20(7,45)$ & $15(6,31)$ & $33(22,45)$ & $1(0,9)$ & $0(.,)$. \\
\hline \multirow[t]{2}{*}{$\begin{array}{l}\text { Meta-analytic } \\
\text { summary }^{b}\end{array}$} & $6(3,13)$ & $11(7,16)$ & $11(9,14)$ & $16(14,19)$ & $19(17,21)$ & $20(17,22)$ & $19(16,24)$ & $23(17,31)$ & $16(11,23)$ & $28(24,31)$ & $21(11,40)$ & $14(7,29)$ \\
\hline & \multicolumn{12}{|c|}{ Panel B. Estimated Transition Probability Among Females (\%) } \\
\hline 2010 & $5(1,17)$ & $7(3,15)$ & $10(5,19)$ & $9(6,13)$ & $14(7,23)$ & $16(9,25)$ & $11(5,20)$ & $5(2,13)$ & $12(5,27)$ & $17(9,29)$ & $15(8,26)$ & $27(5,70)$ \\
\hline 2011 & $12(2,45)$ & $9(4,18)$ & $10(6,18)$ & $18(12,27)$ & $11(7,17)$ & $20(11,32)$ & $14(9,22)$ & $7(2,20)$ & $16(6,35)$ & $24(16,33)$ & $8(1,34)$ & $0(.,)$. \\
\hline 2012 & $16(5,41)$ & $18(5,47)$ & $10(5,21)$ & $11(7,17)$ & $13(8,20)$ & $15(8,25)$ & $14(8,24)$ & $12(5,26)$ & $4(1,12)$ & $18(12,26)$ & $2(0,14)$ & $0(.,)$. \\
\hline 2013 & $9(2,39)$ & $5(2,12)$ & $6(3,12)$ & $11(7,18)$ & $12(6,20)$ & $12(7,20)$ & $11(5,24)$ & $11(4,23)$ & $9(3,21)$ & $14(8,22)$ & $0(.,)$. & $0(.,)$. \\
\hline 2014 & $3(0,18)$ & $15(7,32)$ & $9(4,18)$ & $14(8,22)$ & $19(11,31)$ & $19(11,31)$ & $19(11,31)$ & $15(8,28)$ & $10(3,28)$ & $18(11,29)$ & $40(7,86)$ & $13(2,57)$ \\
\hline $\begin{array}{c}\text { Meta-analytic } \\
\text { summary }^{\mathrm{b}}\end{array}$ & $9(6,13)$ & $11(9,14)$ & $11(9,13)$ & $13(11,15)$ & $13(11,15)$ & $14(11,18)$ & $15(12,17)$ & $11(8,14)$ & $11(8,14)$ & $18(15,21)$ & $12(6,25)$ & $21(12,35)$ \\
\hline
\end{tabular}


b. Meta-analysis summary estimates with each year treated as an independent replication. For 12-, 13-, 18-, 19-, 20-, and 21-year old males as well as 17- and 22year-old females, heterogeneity across replications motivated use of the random effects variance estimation approach. 